\title{
STOCHASTIC ANALYSIS IN THE ACOUSTICS OF DAMPED SOUNDS
}

\begin{abstract}
A stochastic model of sound propagation in damping medium is proposed. It consists of: (1) Ito's stochastic differential equation describing the sound propagation, (2) a potential which models the damping effects. However, due to presence of path integrals this model is elaborate and time consuming, hence inappropriate for numerical simulations and/or model calibrations. To make it simpler we usde the classical results of stochastic analysis; Feynman-Kac formula and Girsanov tansformation obtaining easy-to-use computational procedure for practical purposes.
\end{abstract}

Keywords: Acoustic signal processing, Feynman-Kac formula, Acoustic statistical characteristic, Stochastic processes

\section{ANALIZA STOCHASTYCZNA W BADANIACH PROPAGACJI DŹWIĘKU W OŚRODKACH TŁUMIACYCH}

W pracy przedstawiono stochastyczny model propagacji dźwięku w ośrodku ttumiacym. W badaniach, do modelowania propagacji dźwięku, zastosowano stochastyczne równanie rózniczkowe Ito. Zjawiska ttumienia zamodelowano z zastosowaniem potencjału V(x). Ze względu na obecność całki po trajektoriach opracowanie modelu jest czasochtonne. W celu uproszczenia modelu $i$ umożliwienia jego zastosowania w symulacjach numerycznych wykorzystano wyniki klasycznej analizy stochastycznej. Zastosowanie wzoru Feynmana-Kaca i transformacji Grisanova umożliwiło opracowanie łatwej w użyciu procedury obliczeniowej do zastosowań praktycznych.

Stowa kluczowe: przetwarzanie sygnatów akustycznych, wzór Feynmana-Kaca, procesy stochastyczne

\section{INTRODUCTION}

Sound propagation is a complex phenomenon. Spatial sources locations and medium damping properties have a strong influence on the whole process. In this paper we are going to study only the quantitative aspects of the simplest version of the problem - one source and one receiver. Our goal is to find a mathematical model and easy-to-use numerical procedure. In order to describe sound propagation we apply Ito's stochastic differential equation (SDE). Although these types of equations are rudimentarily used to model diffusion processes rather than sound propagation phenomenons, we find in the mathematical literature attempts to extend this tool to wave phenomenons (Bakhtin and Mueller 2010, Dalang, Mueller and Tribe 2008, Pal and Shkolnikov 2013, Chatterejee 2013). Beside of Ito's equation an integral part of the model is a potential function $V(x)$ in the space variables $x$, which is responsible for the damping effect. However, this effect depends on the whole trajectory of $x(t)$, a solution of Ito's equation and values $V(x(t))$ of the potential on the observation interval $[0, T]$. In the result any calculation on the model, or simulations of the damping effect must be onerous and time consuming. The purpose of this short note is to eliminate this defect. In order to achieve this goal we apply two classical tools of stochastic analysis; the Feynman-Kac formula and Girsanov transformation. In this way we are able to simplify the procedure since the resulting formulae do not include path-integrations.

The paper is organized as follows. In the two subsequent sections we introduce the model, quote the Feynman-Kac and Girsanov theorems. In section 4 we show how these tools could be used to eliminate the path integration at the extra cost of solving an ordinary differential equation of the low order. In the section 5 we explain how to transform our model into stepby-step a procedure leading to practical calculations and/or model calibration.

\section{THE MODEL}

Let us consider the simplest experiment of sounds propagation consisting with one source and one receiver, the both located in the fixed, different points of space $S$ and $R$. Assume that sound volume level (SVL) is observed and measured at some fixed point can be described by Ito's equation (Karataz and Shreve 1991, chapter 5)

$$
X(t)=x+\int_{s}^{t} f(X(r)) d r+\int_{s}^{t} g(X(r)) d W(r) .
$$

In theory of diffusion the quantity $X(t)$ describes the position of single particle at time instant. If the particles are absorbed by the medium, then a special function $V(x)$, called a potential is introduced. According to the general theory of diffusion $V(X(t))$ is the rate of 'killing' the particles at the position $X(t)$ (Ito and McKean 1970, chapter 5). Since $V(x)$ acts similarly as a dissipative force, we adopt it as a model of damping factor. Indeed, in diffusion processes the potential $V(x)$ has a strong influence on any aspect of $X(t)$ behavior modifying the effect of $f(x)$ and $g(x)$. Additionally, $V(x)$ decreases the probability total mass as the effect of killing. The action of $V(x)$ on $X(t)$ is described by the famous Feynman-Kac theorem (Karataz and Shreve 1991, chapter 5, theorem 7.6).

Theorem 1. Let (a) $h: \mathbb{R} \rightarrow \mathbb{R}$ satisfies polynomial growth condition, i.e., $|h(x)| \leq M\left(1+|x|^{n}\right), n \geq 1$, (b) $V: \mathbb{R} \rightarrow[0, \infty)$, (c) $f, g$ are globally Lipschitz, i.e., $|f(x)-f(y)| \leq M|x-y|$.

* Lublin Technical University, Department of Quantitative Methods in Management, Lublin, Poland, e-mail t.banek@pollub.pl 
Then (!) (1) has unique strong solution $X(t), t \in[0, T]$ for any initial condition $X(s)=x$, (!!) the solution $u$ of the equation

$$
\left(\partial_{s}+\mathscr{A}+V\right) u(s, x)=0
$$

where

$$
\mathscr{A} \equiv \frac{g^{2}(x)}{2} \partial_{x x}^{2}+f(x) \partial_{x}
$$

with the Cauchy condition $u(T, x)=h(x)$, is of $C^{1,2}([0, T], \mathbb{R})$ class and is given by the formula

$$
u(s, x)=\mathbb{E}_{\mathbb{P}}\left[h(X(T)) \exp \int_{s}^{T} V(X(t)) d t\right]
$$

where $\mathbb{E}_{\mathbb{P}}$ denotes expectation with respect to the probability measure $\mathbb{P},(\Omega, \digamma, \mathbb{P})$ is a probability space where all stochastic objects, i.e., the processes $W(t), X(t)$ are defined.

Remark 2. If $h$ is a characteristic function of fixed interval $[a, b]$, i.e., $h(x)=1$ if $x \in[a, b]$, and $h(x)=0$ if $x \notin[a, b]$, then

$$
u(s, x)=\mathbb{P}[X(T) \in[a, b]]
$$

Remark 3. In our model (4) gives a probability that the value of damped sound is included in $[a, b]$.

Remark 4. As was mentioned in the introduction, application of the formula (3) is elaborate and time consuming. Any simulation must operate on the whole sampled trajectory of $X(t)$ because of the path integral in the exponent of the right hand side of (3). This makes the formula useless for practical purposes. In the case when additionally the model characteristics, i.e., $f(x), g(x), V(x)$, are uncertain and are known only with some error, hence many simulations are needed, the model seems to be useless too. However, in the next section we introduce a second tool, Girsanov transformation, which allows to eliminate the path integrations in (3).

\section{MEASURE TRANSFORMATION}

Measure transformation is a powerful method in Probability Theory. We introduce now a particularly important example known as Girsanov theorem (Karataz and Shreve 1991, chapter 3 , theorem 5.1). For a function $\varkappa: \mathbb{R} \rightarrow \mathbb{R}$, let define a new process $w(t)$ on $(\Omega, \digamma, \mathbb{P})$ given by the formula

$$
w(t)=W(t)-W(s)-\int_{s}^{t} \varkappa(X(r)) d r
$$

and a set function

$$
\mathbb{Q}(A)=\int_{A} \Lambda_{s}^{T} d \mathbb{P}
$$

for $A \in \digamma$, where for $0 \leq s<T$

$$
\Lambda_{s}^{T}=\exp \left[\int_{s}^{T} \varkappa(X(t)) d W(t)-\frac{1}{2} \int_{s}^{T} \varkappa^{2}(X(t)) d t\right] .
$$

Theorem 5. (Girsanov) Let $\varkappa$ be a continuous function $\varkappa \in$ $C(\mathbb{R})$ with linear growth $|\varkappa(x)| \leq M(1+|x|)$. Then $\mathbb{Q}$ is a probability measure on $(\Omega, \digamma)$, and $w(t)$ given by (5) is a Wiener process with respect to $\mathbb{Q}$. The process $X(t)$ on the new probability space $(\Omega, \digamma, \mathbb{Q})$ satisfies the equation

$$
\begin{aligned}
X(t)=x & +\int_{s}^{t}[f(X(r))+g(X(r)) \varkappa(X(r))] d r \\
& +\int_{s}^{t} g(X(r)) d w(r) .
\end{aligned}
$$

\section{APPLICATION}

In order to apply the Girsanov theorem to the formula (3), we shall use Ito's formula to remove the stochastic integral in (7). Assume, that $K \in C^{2}(\mathbb{R})$ satisfies

$$
g(x) K^{\prime}(x)=\varkappa(x) .
$$

Then from Ito's formula

$$
\begin{aligned}
\int_{s}^{T} \varkappa(X(t)) d W(t)= & K(X(T))-K(X(s)) \\
& -\int_{s}^{T} \mathscr{A} K(X(t)) d t
\end{aligned}
$$

and consequently (7) now takes the form

$$
\begin{aligned}
\Lambda_{s}^{T}= & \exp \{K(X(T))-K(x) \\
& \left.-\int_{s}^{T}\left[\mathscr{A} K(X(t))+\frac{1}{2} \varkappa^{2}(X(t))\right] d t\right\} .
\end{aligned}
$$

Conclusion 6. For any $H: \mathbb{R} \rightarrow \mathbb{R}$ satisfying polynomial growth condition, we have

$$
\begin{aligned}
\mathbb{E}_{\mathbb{Q}}[H(X(T))] & \\
=\mathbb{E}_{\mathbb{P}}\left[H(X(T)) \Lambda_{s}^{T}\right] & \mathbb{E}_{\mathbb{P}}[H(X(T)) \exp \{K(X(T))-K(x) \\
& \left.\left.\quad-\int_{s}^{T}\left(\mathscr{A} K(X(t))+\frac{1}{2} \varkappa^{2}(X(t))\right) d t\right\}\right] \\
=\exp [-K(x)] \mathbb{E}_{\mathbb{P}}\left[\phi ( X ( T ) ) \operatorname { e x p } \left\{-\int_{s}^{T}(\mathscr{A} K(X(t))\right.\right. & \left.\left.\left.\quad+\frac{1}{2} \varkappa^{2}(X(t))\right) d t\right\}\right],
\end{aligned}
$$

where $\mathbb{E}_{\mathbb{Q}}$ denotes expectation with respect to the measure $\mathbb{Q}$, and

$$
\phi(x)=H(x) \exp K(x) .
$$

Conclusion 7. If $K$ solves ordinary differential equation

$$
\mathscr{A} K(x)+\frac{1}{2}\left[g(x) K^{\prime}(x)\right]^{2}+V(x)=0,
$$

then

$$
\begin{aligned}
& \mathbb{E}_{\mathbb{P}}\left[\phi(X(T)) \exp \int_{s}^{T} V(X(t)) d t\right] \\
= & \exp [K(x)] \mathbb{E}_{\mathbb{Q}}[H(X(T))] .
\end{aligned}
$$


Theorem 8. If $K(x) \in C^{2}(\mathbb{R})$ satisfies (15), is nonnegative $(K(x) \geq 0)$, or $K(x)$ has polynomial growth, then

$$
\begin{aligned}
& \mathbb{E}_{\mathbb{P}}\left[h(X(T)) \exp \int_{s}^{T} V(X(t)) d t\right] \\
= & \exp [K(x)] \mathbb{E}_{\mathbb{Q}}[h(X(T)) \exp [-K(X(T))]] .
\end{aligned}
$$

Proof. Put

$$
H(x)=h(x) \exp [-K(x)]
$$

Now

$$
\begin{aligned}
& \mathbb{E}_{\mathbb{P}}\left[h(X(T)) \exp \int_{s}^{T} V(X(t)) d t\right] \text { from (13)(18) } \\
= & \mathbb{E}_{\mathbb{P}}\left[H(X(T)) \exp K(X(T)) \int_{s}^{T} V(X(t)) d t\right] \text { from }(15) \\
= & \mathbb{E}_{\mathbb{P}}[H(X(T)) \exp K(X(T)) \\
& \left.-\int_{s}^{T}\left(\mathscr{A} K(X(t))+\frac{1}{2} \varkappa^{2}(X(t))\right) d t\right] \text { from }(1) \\
= & \exp [K(x)] \mathbb{E}_{\mathbb{P}}\left[H(X(T)) \Lambda_{s}^{T}\right] \text { from }(12) \\
= & \exp [K(x)] \mathbb{E}_{\mathbb{Q}}[H(X(T))] \\
= & \exp [K(x)] \mathbb{E}_{\mathbb{Q}}[h(X(T)) \exp [-K(X(T))]]
\end{aligned}
$$

The assumptions on $K(x)$ are needed to guarantee the existence of the integral in the right hand side of the last equality.

Remark 9. In the last theorem the elimination of the path space integration promised in the Introduction takes place. Indeed, this integration in the left of (17) is annihilated and does not appear in the right. The expectation on the left applies to the process influenced by the potential $V(x)$. Transforming the initial measure $\mathbb{P}$ into a new measure $\mathbb{Q}$ we get an equivalent expression in which the path integral is absent, i.e., process $X(t)$ under the new measure is influenced by $V(x) \equiv 0$. Moreover, the expectation on the right of (17) must be computed at the final time $T$ only, what makes any simulations or numerical calculations much easier. However, in order to do so one must solve the ordinary differential equation (15), getting $K(x)$ for given $V(x)$. Next, from (9) we have $\varkappa(x)$ what allows to define the right hand side of $(8)$ which describes the evolution of $X(t)$, and the value of $X(T)$. The simulated values of $X(T)$ can be substituted in the right of (17). Concluding one may say, that at a small price of the process (1) complication we replace the path space averaging in the left of (17), with averaging the values of modified process, but taken at the final time only.

\section{PROCEDURE IN STEPS}

We describe now a procedure which systematizes the results of the previous sections making them a machinery for numerical computations of $u(s, x)$ given in (3).
Step 1. Given $f, g, V$, find $K(x)$ satisfying

$$
\frac{g^{2}(x)}{2} K^{\prime \prime}(x)+f(x) K^{\prime}(x)+\frac{1}{2}\left[g(x) K^{\prime}(x)\right]^{2}+V(x)=0 .
$$

Step 2. Simulate $X(T)$ given by

$$
\begin{aligned}
X(T)=x & +\int_{s}^{T}\left(f(X(t))+g^{2}(X(t)) K^{\prime}(X(t))\right) d t \\
& +\int_{s}^{T} g(X(t)) d w(t)
\end{aligned}
$$

for some Wiener process $w(t)$, and $K(x)$ obtained in Step 1. Denote $X_{i}(T)$ the value of $i$-th simulation. The stochastic integral approximate by finite sums

$$
\sum_{j \in N} g\left(X\left(t_{j}\right)\right)\left[w\left(t_{j+1}\right)-w\left(t_{j}\right)\right]
$$

Step 3. Substitute the values $X_{i}(T)$ into $h(x)$, and $\exp [-K(x)]$ getting the sets $\left\{h\left(X_{i}(T)\right)\right\}$ and $\left\{\exp \left[-K\left(X_{i}(T)\right)\right]\right\}$.

Step 4. Average the product $h(X(T)) \exp [-K(X(T))]$ with respect to $\mathbb{Q}$, i.e, calculate

$$
\begin{aligned}
\mathbb{E}_{\mathbb{Q}} & {[h(X(T)) \exp [-K(X(T))]] } \\
& \approx \sum_{i \in N} h\left(X_{i}(T)\right) \exp \left[-K\left(X_{i}(T)\right)\right] / N
\end{aligned}
$$

\section{FINAL REMARKS}

In his section we recall some notions of acoustic analysis used by practitioners and indicate the counterparts in our model. Let $P$ denotes acoustic pressure, $P_{0}$ reference pressure, $L$ sound level measured in a fixed space point is defined in formula

$$
L=10 \log \frac{1}{\Delta} \int_{\Delta} \frac{P^{2}(t)}{P_{0}^{2}} d t
$$

where $\Delta$ is an interval of averaging. If $\Delta=[t-\delta, t]$, then for the moving average $L(t)$ we have

$$
L(t)=10 \log \frac{1}{\delta} \int_{t-\delta}^{t} \frac{P^{2}(s)}{P_{0}^{2}} d s
$$

which for $\delta$ close to zero, behaves similarly to the trajectories of $X(t)$ described in section 2 . Let $L_{i}, i=1, \ldots, n$, are values of $L$ computed for $i$-th observation in a fixed point of acoustic space. Then, a common practice in acoustics is to define the acoustic average $L_{A}$ by formula

$$
L_{A}=10 \log \frac{1}{n} \sum_{i=1}^{n} 10^{L_{i} / 10}
$$

and consequently, for moving $L(t)$, the acoustic moving average $L_{A}(t)$ by

$$
L_{A}(t)=10 \log \frac{1}{n} \sum_{i=1}^{n} 10^{L_{i}(t) / 10} .
$$


Two important properties of this kind of averaging should be mentioned. First, there is no need to known the true probability distribution functions (pdf) of $L_{i}$ for $L_{A}$ to compute, and second, this averaging is not additive. If the first thing has some advantages over the averaging based on the pdf, specially when a date set is small, then the second makes difficulties when the date set is growing. In particular, the non-additivity makes recurrence computing practically unavailable what is in sharp contrast with probability methods.

The averaging in our model is certainly additive and formula (19) is convenient in calculations for samples of any size. Recurrence formulae are also easily available.

\section{References}

Bakhtin Y., Mueller C., 2010, Solutions of semilinear wave equation via stochastic cascades. Commun. Stoch. Anal. 4, No. 3, 425-431.

Chatterjee S., 2013, http://arxiv.org/pdf/1306.2382.pdf.

Dalang R., Mueller C., Tribe R. 2008, A Feynman-Kac-type formula for the deterministic and stochastic wave equations and other p.d.e.'s. Trans. Amer. Math. Soc. 360, No. 9, 4681-4703.

Ito K., McKean H.P. Jr, 1970, Diffusion processes and their sample paths. Springer.

Karatzas I., Shreve S.E., 1991, Brownian Mation and Stochastic Calculus. Springer-Verlag.

Pal S., Shkolnikov M., 2013, http://arxiv.org/abs/1306.0857. 